\title{
A Preprocessing Method for Field Strength of Electromagnetism Spectrum Monitor Signal Based on EMD
}

\author{
Gu Xiaopeng ${ }^{1, a}$, Xie Shuguo ${ }^{2, b}$ and Wang $\mathrm{Chao}^{3, \mathrm{c}}$ \\ 1,2,3School of Electronic and Information Engineering, \\ Beijing University of Aeronautics and Astronautics,Beijing 100191,China \\ a,b,c guxiaopeng@buaa.edu.cn
}

Keywords: field strength, preprocessing, fading, EMD, electromagnetism spectrum

Abstract. In the mobile field strength test including road testing and so on, because of the complexity of the city electromagnetic environment, the received field strength value is easy to be affected by Fading and therefore the result is not accurate. In this paper, A Preprocessing Method for field strength of electromagnetism spectrum monitor signal based on EMD(Empirical Mode Decomposition) is proposed. It is aimed at the slow change characteristics of adjacent field strength in the mobile field strength test. Through the adaptive decomposition of the field strength value series by using EMD, it eliminates the fading and gets more accurate field strength value. The validity of the method is demonstrated by simulation and experiment.

\section{Introduction}

The road test is a common method to test the wireless signal in the communication industry[1]. Through the test, the relevant data of the electromagnetic spectrum signal is collected along the way[2]. It can accurately understand the field strength coverage and other information of the test area, and used to support the reasonable planning and optimization of the electromagnetic environment[3]. ITU(International Telecommunication Union) is also proposed to monitor the field strength in path measure to predict the regional field strength and so on[4]. In addition, the mobile monitoring can be used to locate the radiation source in the area by the field strength fading model. In these applications, the accuracy of the received field strength determines the test results[5]. The method proposed in this paper is aimed at adaptive processing of the received field strength, and get more accurate field strength values. Lay the foundation for the good results of mobile monitoring.

\section{Processing Model}

Today, the electromagnetic environment is becoming increasingly complex. In this environment, the received signal strength will be affected by fast fading and slow fading. This effect leads to a large deviation between the received field strength values and the actual field strength values. Among them, the fast fading is the fast jitter of the signal, which is caused by the multipath effect. It is generally fits Rayleigh distribution. Slow fading is the shadow fading, which is caused by the emission, absorption and scattering of the obstacles[6]. It is generally fits Normal distribution. As shown in the figure 1:

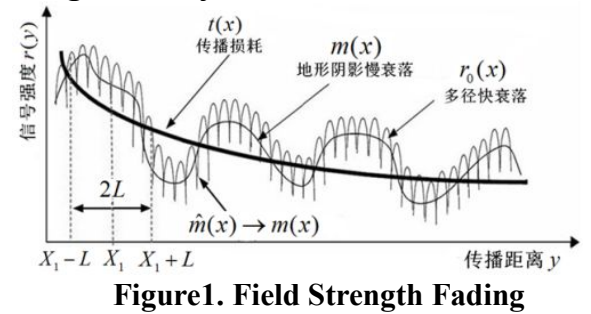

William C. Y. Lee proposed in the literature that the signal received by the mobile platform can be expressed as the following formula:

$$
\mathrm{r}(\mathrm{x})=\mathrm{m}(\mathrm{x}) \cdot r_{0}(\mathrm{x})
$$


Among them, $\mathrm{x}$ is the distance; $\mathrm{r}(\mathrm{x})$ is the receiving signal; $r_{0}(\mathrm{x})$ is Rayleigh fading, which is fast fading; $\mathrm{m}(\mathrm{x})$ is the local mean, which is the synthesis of slow fading and spatial transmission loss, and it can be expressed as:

$$
\mathrm{m}(\mathrm{x})=\frac{1}{2 L} \int_{x-L}^{x+L} r(y) d y
$$

Among them, $2 \mathrm{~L}$ is the average sampling interval length, also called the intrinsic length. William C. Y. Lee pointed out that it can effectively achieve the purpose of eliminating the fast fading, when the intrinsic length is 40 wavelengths, sampling 36- 50 sample points[7]. This is usually the preprocessing method. However, due to the slow fading caused by terrain ups and downs, building block and so on, there is still a big difference between the actual field strength and the received signal strength.

By the formula, it can be seen that the field strength values of mobile monitoring are only related to the propagation loss, when the fast fading and slow fading are eliminated, and the adjacent field strength is slowly changed[8]. So the complex nonlinear components in the envelope of the received field strength value can be considered as noise, then de-noise by the method. It can effectively remove the fading, and the field strength value series has the higher credibility and availability.

In de-noising method, the commonly used fourier transform is only for stationary signals and it is more practical when the spectral characteristics of the noise are different from the spectral characteristics of the signal. The non-stationary signal is regarded as the superposition of a series of short-time stationary signals by window fourier transform. It can be achieved by adding window and moving window in time domain, but it lacks adaptability, and its resolution is single.

The wavelet transform overcomes the deficiency of constant window size of window Fourier transform. It can be sensitive to the change of the signal. The multi - resolution can make the effective components and noise in the non-stationary signal to be different characteristics. However, the selection of wavelet base has a great effect on the de-noising, and the prior information is needed to improve the de-noising effect. So the method based on wavelet transform can solve the problem, but it is not adaptive[9].

In 1998, N. E. Huang et al proposed a non-stationary signal decomposition method: EMD[10]. It decomposes the complex signal into a number of IMF in accordance with the frequency from high to low. The difference between this method and wavelet analysis is that it is a posteriori, and it is not necessary to select the basis function in advance. It is adaptive to produce a suitable mode function according to the characteristics of the signal[11]. After the EMD decomposition, the IMF component is arranged according to the frequency from high to low. According to this characteristic, to remove a number of high-frequency IMF components and reconstruct the sequence with other components, which equivalent to keep the slow changes in adjacent field strength value and eliminate the influence of fast fading and slow fading.

Specific method of noise elimination: find the maximum value of the sequence ${ }^{x(t)}$, and fit the maximum envelope by interpolation; Similarly, find the minimum value of the sequence $x(t)$, and fit the minimum envelope by interpolation. The mean value of the upper and lower envelope is used as the mean envelope of the sequence $m(t)$. Then decompose the first Intrinsic Mode Function $c_{1}(t)$ by using the original time series ${ }^{x(t)}$ minus the mean of the envelope ${ }^{m(t)}$. Using ${ }^{x(t)}$ minus $c_{1}(t)$, the remaining value sequences are obtained:

$$
x_{1}(t)=x(t)-c_{1}(t)
$$

Take ${ }^{x_{1}(t)}$ as a new "original sequence". Then repeat the above steps until the nth Intrinsic Mode Function is extracted. The last remaining remainder is $r_{n}(t)$. Therefore:

$$
x^{s}(t)=c_{\mathrm{n}}(t)+r_{n}(t)
$$

The formula (4) is a series of field strength value after noise elimination. Decomposition process as shown in Figure 2 


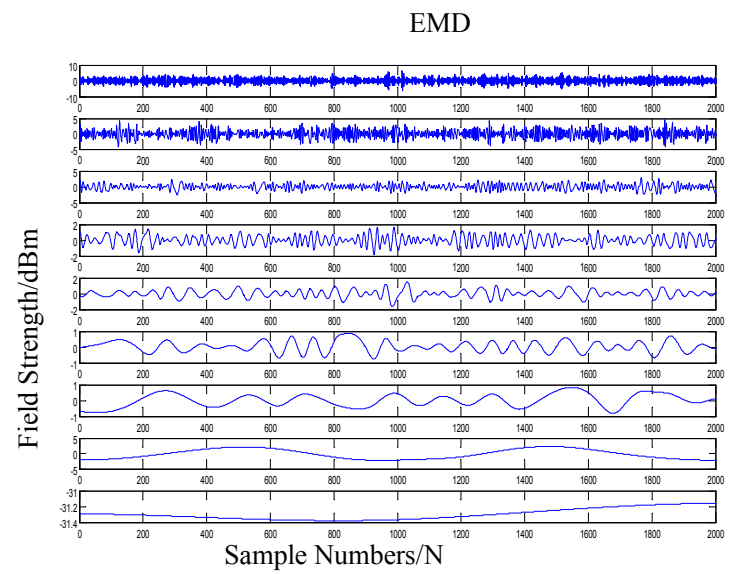

Figure2. EMD Decomposition Process

\section{Method Verification}

Verification by Simulation. Assuming the coordinate axis X from 0 to 1000 , y from 0 to 1000 for the monitoring area, the unit of the coordinates is $\mathrm{m}$. There are a frequency sources, the frequency is $800 \mathrm{MHz}$. The sources is located on the axis of $\mathrm{A}(500,500)$, the power is $60 \mathrm{dBm}$. The height of the source is $30 \mathrm{~m}$. Receiving antenna height is $2 \mathrm{~m}$.

Assuming that the mobile monitoring system receives 10 sets of field strength data and corresponding coordinate position information per second. Start to move and monitor from the coordinates $(0,1000)$ with uniform speed $10 \mathrm{~m} / \mathrm{s}$. Move to the coordinates $(1000,1000)$ along the positive direction of the $\mathrm{X}$ axis, and then move to the coordinates $(1000,0)$ along the negative direction of the $\mathrm{Y}$ axis. A total of 2000 sets of field strength and coordinate position information can be received, 1 sets of data per 1 meters. Using the Egli wave propagation model to simulate the field strength value on the point of the moving path, and the received field strength sequence in different position is imitated by simulation of the multipath fading noise and shadow slow fading noise.

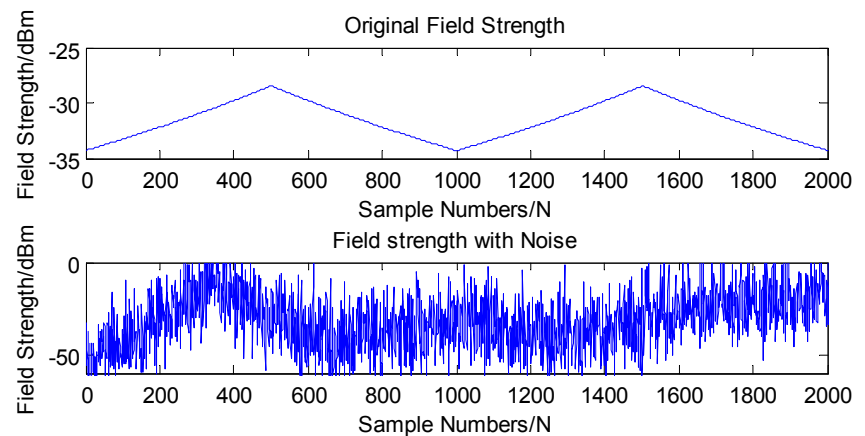

Figure3. Original Field Strength and Field Strength with Noise

Through the Figure3, it can be seen that the received field strength value after fading superposition is basically covered, and can not be used directly.

Using the field strength median method, wavelet de-noising and the EMD to preprocess the received field strength series by fading superposition respectively.

The results of field strength median method are shown in Figure 4.

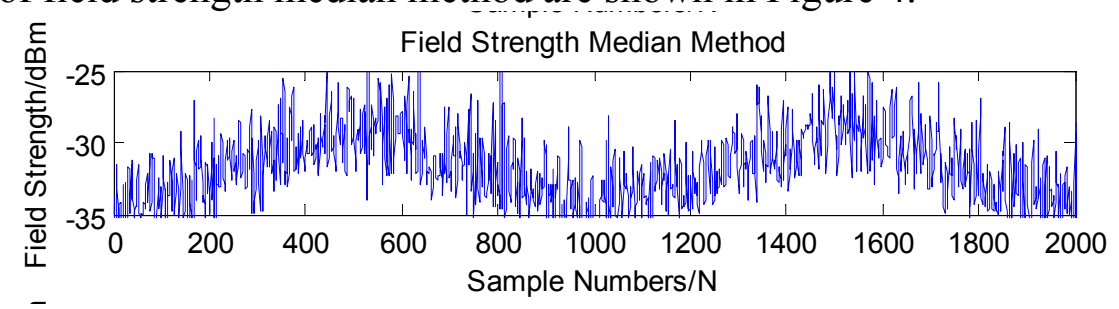

Figure4. The Results of Field Strength Median Method 
It can be seen that the field strength median method removes some noise, but it still has a big difference with the real field strength value.

Use wavelet Mallat algorithm for analysis in wavelet de-noising method. Through many experiments, the dB5 wavelet for 7 level decomposition is chosen. Use the method of wavelet de-noising method to deal with the field strength series, which treated by the field strength median method. The result is shown in Figure 5.

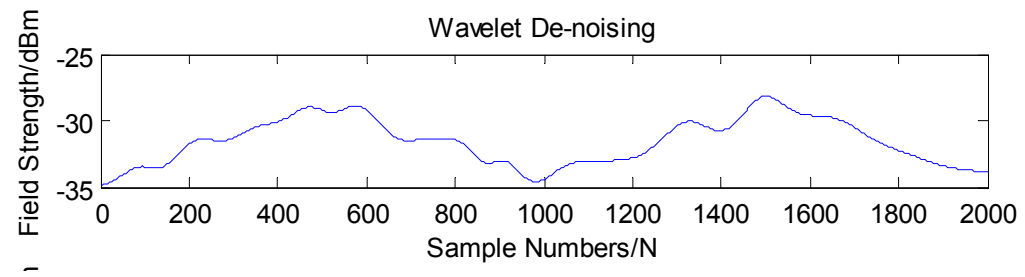

Figure5. The Results of Wavelet De-noising

It can be seen that the noise caused by the fading is basically eliminated. The available field strength series are obtained.

Then, use the EMD to preprocess the field strength series. The result is shown in Figure 6.

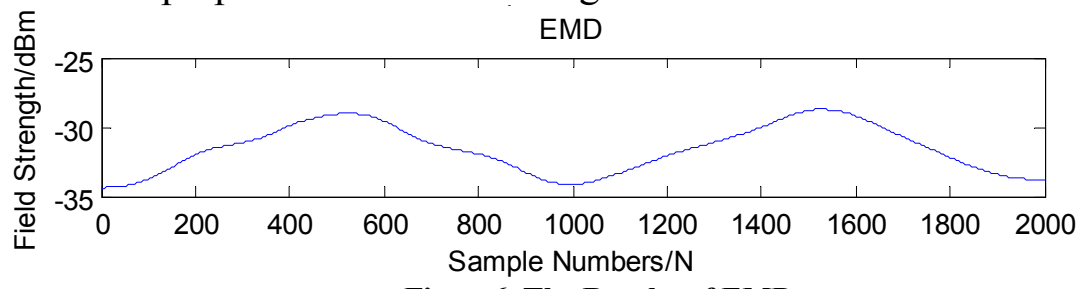

Figure6. The Results of EMD

The result shows that the field strength series after pretreatment by the EMD are basically eliminated the noise, and it does not need to adjust the parameters in advance. After comparison, the deviation mean between the field strength series after pretreatment and the original field strength series is $0.721 \mathrm{dBm}$, the standard deviation is 0.534

It can be seen that the EMD method proposed in this paper can be adaptive to eliminate the noise caused by the fading, and the effect is good.

Verification by Experiments. Use two same frequency source to transmit $450 \mathrm{MHz}$ signal in the external field. Use the mobile field strength monitoring system composed of USRP (Universal Software Radio Peripheral), GPS and computer to receive and process signals. Among them, USRP is used as the receiving device of the field strength, GPS is used as the equipment for measuring the position of its own, and the computer is used to process and calculate the field strength data and position data received. Preprocess the received field strength sequence by EMD, the results are shown in Figure 7:
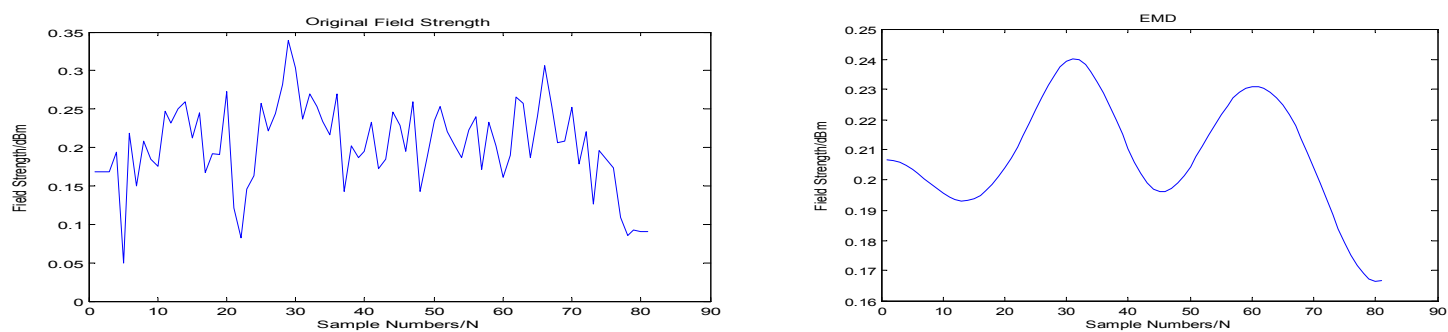

Figure7. The Original Field Strength and Preprocess based on EMD

Use the field strength sequences before and after processed respectively and location method based on field strength for localization of source, the results are shown in Figure8 . 

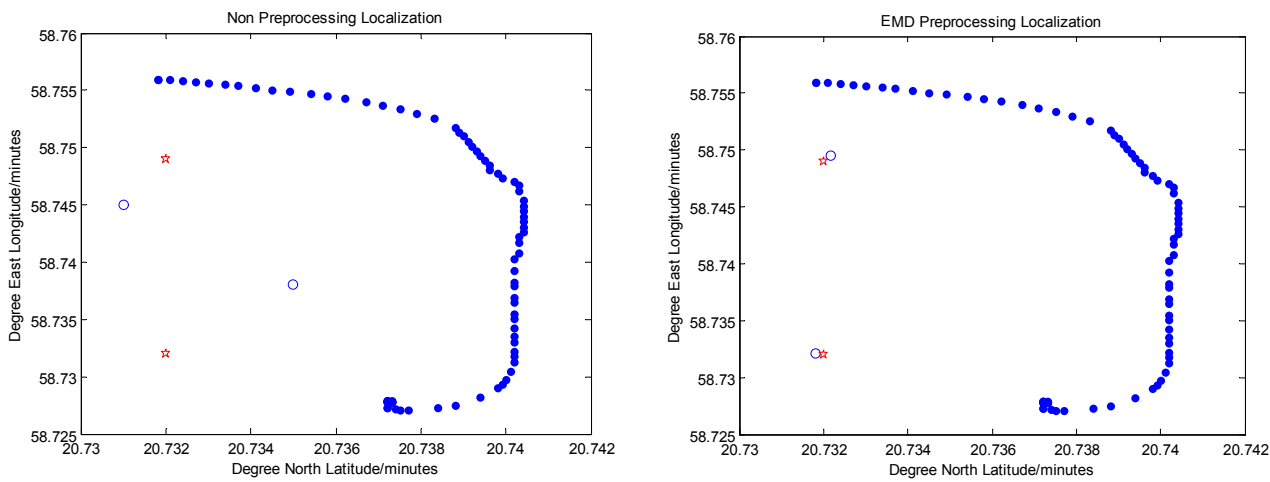

Figure8. The Localization Results of Non Preprocessing and based on EMD

It can be seen in the same measurement data and the same locating method, the locating error processed by EMD is smaller. This shows that the method proposed in this paper is more effective.

\section{Conclusion}

In this paper, we propose a preprocessing Method for field strength of electromagnetism spectrum monitor signal based on EMD, which can be adaptive to eliminate the noise caused by fast fading and slow fading. It can provide a great help for the further utilization of the measurement value, because of a good reduction of the received field strength value. It has a significant application value.

\section{Reference}

[1] Bertoni H L. Radio Propagation for Modern Wireless Systems[J]. Prentice Hall Ptr, 2000.

[2] LI Ru xin, WANG Dao heng, XU Ji sheng, et al. The Research for Field Intensity Prediction Software of Radio Signals in Mobile Networks[J]. Tianjin Communications Technology, 2003.

[3] D. Gabor. Theory of communications [J]. Journal Institute of Electrical Engineers, 1946, 93: 429-457.

[4] Bertoni H L. Coverage prediction for mobile radio systems operating in the $800 / 900 \mathrm{MHz}$ frequency range[J]. Vehicular Technology IEEE Transactions on, 1988, 37(1):3 - 72.

[5] Wu Xiaoping Pang Qinhua. Research on Mobile Station Locating Algorithm by the signal Attenuation Method within Cellular Network[J]. Journal of Beijing University of Posts \& Telecommunications, 1998.

[6] YANG Jun an, ZHUANG Zhen quan, ZHONG Zi fa, et al. A Novel Method Based on GIS and Signal Strength Measurement to Estimate Radiate Source's Location[J]. Journal of University of Science \& Technology of China, 2002.

[7] Lee, W.C.Y. Mobile Communications Engineering [M].McGraw Hill, New York, 1985

[8] Xie S, Zhiqiu Y E, Wang L, et al. Radio source locating method based on signal strength Measurement[J]. Chinese Journal of Radio Science, 2013.

[9] David L. Donoho, Iain M. Johnstone. Adapting to unknown smoothness via wavelet shrinkage[J]. Journal of the American Statistical Association, 1994, 90(432):1200-1224.

[10]Huang N E, Shen Z, Long S R, et al. The empirical mode decomposition and Hilbert spectrum for nonlinear and non-stationary time series analysis [J]. Proc Roy Soc London A, 1998, 454: 903-995.

[11]Huang N E, Shen Z, Long S R. A new view of nonlinear water waves: the Hilbert spectrum[J]. Annual Review of Fluid Mechanics, 2003, 31(1):417-457. 